\title{
Evidencia seroepidemiológica de exposición humana a Anaplasma sp en Santiago, Chile
}

\author{
Katia Abarca V., Javier López Del P., Patricia González A., Jeannette Dabanch P., \\ Marisa Torres H., Verónica Solari G. y Cecilia Perret P.
}

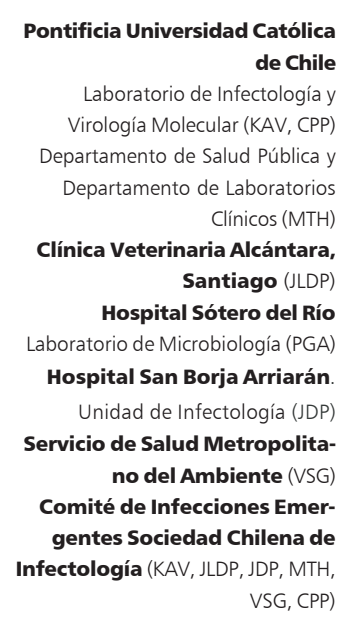

Recibido: 3 enero 2008 Aceptado: 20 junio 2008

Correspondencia a: Katia Abarca Villaseca katia@med.puc.cl

\section{Introducción}

$\mathrm{L}$ a ehrlichiosis/anaplasmosis es una zoonosis emergente reconocida por primera vez en humanos en 1987 en Estados Unidos de Norteamérica (E.U.A.) y luego en Europa. La familia Anaplasmataceae, orden Rickettsiales incluye los géneros Ehrlichia, Anaplasma, Cowdria y Neorickettsia ${ }^{1}$. Las especies de los géneros Ehrlichia y Anaplasma se transmiten por garrapatas. La ehrlichiosis y anaplasmosis humana son causadas predominantemente por Ehrlichia chaffeensis, agente de la ehrlichiosis monocítica humana (EMH) y por Anaplasma phagocytophilum, agente de la anaplasmosis granulocítica humana (AGH). En E.U.A. se presentan en diversas regiones geográficas, se transmiten por distintas garrapatas y sus principales reservorios naturales son los ciervos, cabras, perros y roedores silvestres ${ }^{2,3}$. Las manifestaciones clínicas en humanos son fiebre, compromiso del estado general, cefalea, mialgias, artralgias y en menor proporción exantema maculopapular. El diagnóstico clínico es difícil debido a lo inespecífico de estos síntomas. Además de éstas, se han descrito otras dos especies capaces de causar infección en humanos: Ehrlichia ewingii, que se ha encontrado en individuos inmunocomprometidos, cuyo reservorio es el perro y Amblyoma americanum su vector ${ }^{4}$, y
Neorickettsia sennetsu, descrita en Asia en la década del 50, posiblemente transmitida a través de la ingesta de peces parasitados por helmintos ${ }^{5}$. Existe además una enorme variedad de otras especies descritas en distintas partes del mundo y que infectan otro tipo de animales.

En América del Sur se ha descrito la presencia de ehrlichiosis/anaplasmosis en humanos mediante estudios serológicos realizados en Argentina y Brasil, sin identificarse la especie involucrada ${ }^{6,7}$. En Venezuela se demostró por primera vez infección por E. canis en un humano asintomático ${ }^{8}$, la que posteriormente fue aislada en perros y en Rhipicephalus sanguineus (garrapata café del perro) ${ }^{9}$ y más recientemente detectada en humanos sintomático ${ }^{10}$. Rhipicephalus sanguineus fue descrita por primera vez en Chile en el año 1974'" siendo actualmente su presencia masiva en los meses de primavera y verano en caninos desde Arica hasta Temuco $^{12}$. En 1999 se reportó por primera vez ehrlichiosis canina (EC) en perros de la comuna de Puente Alto (Santiago) que presentaban hemorragias y trombocitopenia ${ }^{13,14}$. Desde esa fecha, el reconocimiento de la enfermedad en perros ha sido creciente en el país, habiéndose identificado recientemente Anaplasma platys como agente causal de esta enfermedad en caninos $^{15}$.

Estudios de prevalencia de ehrlichiosis/anaplas- 
mosis en humanos han mostrado altas tasas de anticuerpos que reaccionan con $A$. phagocytophilum en sujetos sanos que viven en áreas endémicas de AGH en E.U.A. ${ }^{16,17}$. Debido a las reacciones cruzadas obtenidas en estudios serológicos, se considera que con ellos no es posible asegurar la especie, pudiendo representar anticuerpos generados contra especies relacionadas o incluso, otras aún no caracterizadas ${ }^{18}$.

En Chile, estudios en humanos han detectado presencia de anticuerpos contra Ehrlichia sp en 2 de 19 dueños de perros con ehrlichiosis ${ }^{19}$ y contra Anaplasma sp en 26 de 118 sujetos con contacto laboral con $\operatorname{caninos}^{20}$. A pesar de ser una enfermedad de notificación por laboratorio desde el año 2000, no se han reportado casos clínicos de ehrlichiosis/anaplasmosis en humanos en nuestro país.

Con el propósito de buscar mayor evidencia epidemiológica de la exposición humana a Anaplasma sp en la Región Metropolitana de Chile, se diseñó un estudio cuyos objetivos fueron:

- Determinar la presencia de anticuerpos contra Anaplasma sp en individuos en contacto con un caso de EC (grupo de riesgo).

- Identificar eventuales factores de riesgo asociados a la adquisición de la infección.

- Identificar casos de probable ehrlichiosis/anaplasmosis humana en el grupo de riesgo.

\section{Métodos}

Se diseñó un estudio seroepidemiológico en población humana en contacto con casos de EC en tres comunas de la Región Metropolitana (grupo de riesgo) y en un grupo control.

Población. Se consideró grupo de riesgo a los propietarios de 40 perros con diagnóstico de EC y a personas habitantes de casas colindantes. Se consideró EC a perros con diagnóstico clínico basado en manifestaciones hemorrágicas asociadas a trombocitopenia e infestación por garrapatas, atendidos en cuatro clínicas veterinarias de las comunas de La Granja, Puente Alto y Cerro Navia. Treinta de los 40 perros tenían confirmación serológica realizada en una muestra única de suero. El grupo control estuvo constituido por personas sanas, habitantes de la Región Metropolitana, sin antecedentes de contacto con garrapatas ni con perros con EC.

Encuesta y toma de muestras. Se realizó visita domiciliaria al grupo de riesgo y se aplicó una encuesta para evaluar el grado de exposición a garrapatas y eventuales factores de riesgo para la adquisición de la infección: contacto físico cercano con el perro afectado, como dormir con el animal, bañarlo, sacarle garra- patas; haber sido mordido por garrapata; haber encontrado garrapatas en el cuerpo o ropa. Se consideró infestación domiciliaria por garrapatas al antecedente de presencia de al menos una garrapata en el interior del domicilio. Se consultó además por el antecedente de cuadros febriles de etiología no precisada, en los últimos seis meses, que pudieran indicar la presencia de ehrlichiosis/anaplasmosis clínica. Previa firma de consentimiento informado, se tomó muestra de sangre para estudio serológico a las personas con antecedentes de contacto con garrapatas o contacto habitual con el perro índice; igualmente, a los vecinos que aceptaron participar. Previa firma de consentimiento informado, se tomó muestra de sangre para estudio serológico a las personas del grupo control.

Procedimientos de laboratorio. Se estudió presencia de anticuerpos séricos IgG contra Anaplasma sp mediante inmunofluorescencia indirecta (IFI) (Füller Lab, E.U.A.) en ambos grupos. Brevemente, la técnica consiste en depositar suero diluido en láminas que contienen células HL-60 infectadas con el agente de la AGH (A. phagocytophilum). Los anticuerpos específicos unidos se hacen reaccionar con IgG anti-IgG humana marcada con fluoresceína y la reacción es visualizada en microscopio de IF. Los sueros se analizaron a una dilución de $1 / 64$, punto de corte que recomienda el fabricante. Cada vez que se realizó el ensayo, se incluyeron un control negativo y un control positivo en tres diluciones $(1 / 4,1 / 8$ y 1/16) para comparación de la intensidad de la fluorescencia. El procedimiento se realizó en el Laboratorio de Microbiología del Hospital Sótero del Río, las muestras fueron examinadas en forma ciega y separadamente por un tecnólogo médico y un microbiólogo, ambos con experiencia en la técnica. Considerando la reactividad cruzada descrita para los ensayos serológicos entre las distintas especies de Ehrlichia/Anaplasma ${ }^{21}$, las muestras positivas se catalogaron como con seropositividad a Anaplasma sp.

Registro y análisis de resultados. Se registraron los datos de la encuesta domiciliaria y los resultados serológicos en base de datos Excel. El análisis estadístico se realizó con programa estadístico SPSS 8.0 para Windows. Se comparó la seroprevalencia de anticuerpos anti-Anaplasma sp según el tipo de población (grupo de riesgo: contactos domiciliarios y vecinos; grupo control), la presencia de cada factor de riesgo investigado y las características demográficas de la población estudiada (edad y género), mediante prueba de $\chi^{2}$ y prueba exacta de Fischer.

El estudio fue aprobado por los Comités de Ética de la Dirección de Investigación de la Escuela de Medicina de la Pontificia Universidad Católica de Chile y del Servicio de Salud Metropolitano Sur Oriente, área ad- 


\begin{tabular}{|c|c|c|}
\hline Factor de riesgo & $\mathbf{n}$ & $\%$ \\
\hline Bañar al perro & 79 & 73 \\
\hline Sacar garrapatas al perro & 74 & 68 \\
\hline Dormir con el perro & 45 & 42 \\
\hline Hallazgo de garrapatas en el cuerpo o ropa & 12 & 11 \\
\hline Antecedente de mordedura por garrapatas & 9 & 8 \\
\hline
\end{tabular}

ministrativa donde reside la mayoría de los participantes.

\section{Resultados}

Ingresaron 108 personas al grupo de riesgo: 90 contactos domiciliarios de los perros con EC y 18 habitantes de 13 viviendas vecinas. Sesenta y dos por ciento de los participantes eran de género femenino y sus edades fluctuaban entre 5 y 82 años, con una mediana de 34 años. Ingresaron 61 personas al grupo control, $75 \%$ de género femenino, entre 16 y 63 años de edad, mediana 33 años.

De las 53 casas visitadas, 50 (94\%) presentaban infestación por garrapatas. La totalidad de las viviendas poseía perros como mascota.

La frecuencia de los factores de riesgo investigados se muestra en la Tabla 1, ordenados en frecuencia decreciente. No se encontraron personas con historia de síntomas sugerentes de ehrlichiosis/anaplasmosis.

Se detectó presencia de anticuerpos anti-Anaplasma sp en 20 de los $108(18,5 \%)$ sujetos del grupo de riesgo: $17 / 90(18,8 \%)$ en los contactos domiciliarios y $3 / 18$ $(16,6 \%)$ en los vecinos. Estas diferencias no son significativas. No se encontraron diferencias estadísticamente significativas al comparar la seroprevalencia según factores de riesgo y demográficos.

Dos sujetos del grupo control resultaron positivos $(3,3 \%)$. Las diferencias en seroprevalencia del grupo de riesgo comparado con el grupo control son estadísticamente significativas ( $\mathrm{p}<0,005$ ), OR 6,7 (IC 95\% $1,43-43,8)$.

\section{Discusión}

Los resultados aportan mayor evidencia a la existencia de exposición humana a Anaplasma sp en nuestro medio, situación que ya sugerían los estudios serológicos previos ${ }^{19,20}$. La mayor prevalencia de anticuerpos anti-Anaplasma sp en el grupo de riesgo que en el grupo control, apoya fuertemente que las características que diferencian ambos grupos, como son la convivencia cercana con perros enfermos de EC y habitar ambientes con infestación por $R$. sanguineus son factores asociados a la adquisición humana de este agente y que probablemente esta garrapata es el agente transmisor de esta zoonosis de perros a humanos.

Destaca la elevada infestación domiciliaria por garrapatas, la que alcanza más del 90\% de los hogares visitados. Esta cifra supera lo publicado por Ibarra, quien ya hace algunos años encontró frecuencias de infestación de hasta $50 \%$ de las viviendas en algunas comunas de la Región Metropolitana ${ }^{12}$.

Considerando que, a diferencia de otras especies de garrapatas como Amblyoma sp, la garrapata café del perro sólo ocasionalmente puede morder al ser humano ${ }^{22,23}$, el hallazgo de $8 \%$ de personas con antecedente de mordedura por garrapata en este estudio podría explicarse por la alta infestación encontrada, situación que facilita el acercamiento de este vector al ser humano. También se podría explicar por la hipótesis de que existirían poblaciones de $R$. sanguineus más adaptadas al hombre 22 .

Numerosos estudios han evidenciado una importante variabilidad en el diagnóstico serológico de ehrlichiosis/anaplasmosis entre diferentes laboratorios, técnicas, antígenos utilizados, puntos de corte y criterios de positividad ${ }^{18,21,24}$. Estas dificultades en el diagnóstico serológico se ven aún más complicadas por el elevado número de diferentes especies de Ehrlichia/ Anaplasma y la reactividad cruzada entre ellas e, incluso, con otras especies relacionadas ${ }^{21}$. Por estos motivos, el hallazgo de anticuerpos séricos utilizando como antígeno de detección a $A$. phagocytophilum, no permite concluir que sea ésta la especie infectante. La seropositividad observada al usar esta prueba, se podría explicar por la presencia en Chile de A platys, agente recientemente identificado en casos de $\mathrm{EC}^{15}$, puesto que ambas especies están cercanamente relacionadas y pertenecen al mismo grupo (grupo 1) de la familia Anaplasmataceae ${ }^{1}$. Por tanto, el uso de esta prueba diagnóstica puede ser de utilidad en nuestro medio para estudios de seroprevalencia o para apoyar la sospecha clínica de la enfermedad, tanto en caninos como en humanos. Son necesarios mayores estudios para precisar el o los agentes causantes de estas infecciones en humanos en Chile.

Estos resultados, sumados al reconocimiento en Chile de dos agentes infecciosos transmitidos por garrapatas en caninos: A. platys ${ }^{15}$ y Rickettsia sp $^{27}$, indican que en nuestro medio este vector debe ser considerado como un problema no sólo de medicina veterinaria sino también de salud pública. 
Agradecimientos. A Leila Habash, Analía Cuiza y Marcela Urzúa, quienes colaboraron en la realización de las encuestas y tomas de muestras y a Ángela Leone quien participó en la determinación de anticuerpos.

\section{Resumen}

Objetivos y Método: Con el propósito de buscar mayor evidencia de exposición humana a Anaplasma sp en Chile, se estudiaron 108 personas en contacto con perros con ehrlichiosis canina (EC) (grupo de riesgo) y 61 personas sin antecedente de contacto con garrapatas ni con perros con EC (grupo control). Se aplicó encuesta sobre factores de riesgo e historia de cuadros sugerentes de ehrlichiosis/anaplasmosis al grupo de riesgo. En ambos grupos se determinó presencia de IgG anti-Anaplasma sp. Resultados: Se encontró significativa mayor prevalencia de anticuerpos anti-Anaplasma sp en el grupo de riesgo que en el grupo control $(18,5$ versus $3,3 \%), p<0,005$. No se encontraron factores de riesgo asociados a seropositividad, ni personas con historia sugerente de ehrlichiosis/anaplasmosis clínica. Noventa y cuatro por ciento de las viviendas del grupo de riesgo presentaba infestación por garrapatas. Discusión: Se evidencia mayor riesgo de exposición humana a Anaplasma $\mathrm{sp}$ en personas en contacto cercano con perros con EC y que habitan viviendas con infestación por garrapatas.

\section{Referencias}

1.- Dumler J S, Barbet A F, Bekker C P, Dash G A, Palmer G H, Ray S C, et al. Reorganization of genera in the families Rickettsiaceae and Anaplasmataceae in the order Rickettsiales. Int J Syst Evol Microbiol 2001; 51: 2145-65.

2.- McQuiston J, Paddock C, Holman R, Childs J E. The human ehrlichiosis in the United States. Emerg Infect Dis 1999; 5: 635-42.

3.- Keysary A, Amram L, Keren G, Sthoeger Z, Potasman I, Jacob A, et al. Serologic evidence of human monocytic and granulocytic ehrlichiosis in Israel. Emerg Infect Dis 1999; 5: 775-8.

4.- Buller R S, Arens M, Hmiel S P, Paddock C D, Sumner J W, Rikhisa Y, et al. Ehrlichia ewingii, a newly recognized agent of human ehrlichiosis. N Engl J Med 1999; 341: 148-55.

5.- Misao T, Kobayashi Y. Isolation of etiologic agent from blood, bone marrow, and lymph node of a patient with infectious mononucleosis by using mice. Tokyo Iji Shinshi 1954; 71: 683-6.

6.- Ripoll C M, Remondegui C E, Ordonez G, Arazmendi R, Fusaro H, Hyman M J, et al. Evidence of rickettsial spotted fever and ehrlichial infections in a subtropical territory of Jujuy, Argentina. Am J Trop Med Hyg 1999; 6 (2): 350-4.

7.- Calic S B, Galvão M A M, Bacellar F, Rocha C M B M, Mafra C L, Leite R C, et al. Human ehrlichiosis in Brazil: First suspect cases. Braz J Infect Dis 2004; 8 (3): 259-62.

8.- Pérez M, Rikihisa Y, Wen B. Ehrlichia canis-like agent isolated from a man in Venezuela: antigenic and genetic characterization. J Clin Microbiol 1996; 34: 2133-9.

9.- Unver A, Pérez M, Orellana N, Huang H, Rikihisa Y. Molecular and antigenic comparison of Ehrlichia canis isolates from dogs, ticks and human in Venezuela. J Clin Microbiol 2001; 39: 2788-93.

10.- Pérez M, Bodor M, Zhang CH, Xiong Q, Rikihisa Y. Human infection with Ehrlichia canis accompanied by clinical signs in Venezuela. Ann N Y Acad Sci 2006; 1078: 110-7.

11.- Tagle. Presencia accidental de Riphicephalus sanguineus en un perro de Santiago de Chile. Agricultura Técnica 1976; 36: 137.

12.- Ibarra L, Morales M A, Acuña P. Aspectos demográficos de la población de perros y gatos en la ciudad de Santiago, Chile. Avances en Ciencias Veterinarias 2003; 18: 13-20.

13.- López J. Primeros casos de ehrlichiosis canina detectados en Chile. Boletín de la Sociedad Chilena de Infectología. 1999; 6: 1.

14.- López J, Castillo A, Muñoz M, Hildebrand S. Hallazgo de Ehrlichia canis en Chile, informe preliminar. Arch Med Vet 1999; 31: 211-4.

15.- Abarca K, López J, Perret C, Guerrero J, Godoy P, Veloz A, et al. Anaplasma platys in dogs, Chile. Emerg Infect Dis 2007; 13: 1392-5.

16.- Bakken J S, Goellner P, Van Etten M, Boyle DZ, Swogen OL, Mattson S, et al Seroprevalence of human granulocytic ehrlichiosis among permanent residents of northwestern Wisconsin. Clin Infect Dis 1998; 27: 1491-6.

17.- Agüero-Rosenfeld M E, Donnurumma L, Zentmaier L, Jacob J, Frey M, Noto R, et al. Seroprevalence of antibodies that react with Anaplasma phagocytophila, the agent of human granulocytic ehrlichiosis, in different populations in Westchester County, New York. J Clin Microbiol 2002; 40: 2612-15.

18.- Agüero-Rosenfeld M E. Diagnosis of human granulocytic ehrlichiosis: State of the Art. Vector Borne and Zoonotic Dis 2002; 2: 233-9

19.- López J, Rivera M, Concha J C, Gatica S, Loeffeholz M, Barriga O. Ehrlichiosis humana en Chile, evidencia serológica. Rev Méd Chile 2003; 131: 67-70.

20.- Abarca K, García P, López J, Concha M, Habash L, Garay T, et al. Presencia de anticuerpos anti-Ehrlichia spp. en un grupo de riesgo ocupacional. Libro de Resúmenes XX Congreso Chileno de Infectología, La Serena 5-8 de Noviembre, 2003; resumen CO 32, pág 36 .

21.- Dumler J S, Asanovich K M, Bakken J S, Richter P, Kimsey R, Madigan J E. Serologic cross-reactions among Ehrlichia equi, Ehrlichia phagocytophila and human granulocytic ehrlichia. J Clin Microbiol 1995; 33: 1098-103.

22.- Goddard J. Focus of human parasitism by the brown dog tick, Riphicephalus sanguineus (Acari: Ixodidae). J Med Entomol 1989; 26: 628-9.

23.- Sanogo Y, Parola P, Shpynov S, Camicas J L, Brouqui P, Caruso G, et al. Genetic diversity of bacterial agents detected in ticks removed from asymptomatic patients in northeastern Italy. Ann N Y Acad Sci 2003; 1990: 182-90.

24.- Agüero-Rossenfeld M E, Horowitz H W, Wormser G P, McKenna D F, Nowakowski J, Muñoz J, et al. Human granulocytic ehrlichiosis (HE): a case series from a single medical center in New York State. Ann Intern Med 1996; 125: 904-8.

25.- Arraga-Alvarado C, Palmar M, Parra O, Salas P. Fine structural characterization of Rickettsia-like organism in human platelets from patients with symptoms of ehrlichiosis. J Med Microbiol 1999; 48: 991-7.

26.- Arraga-Alvarado C, Montero-Ojeda M, Bernardoni A, Anderson B, Parra O. Ehrlichiosis humana: reporte del primer caso en Venezuela. Invest Clin 1996; 37: 35-49.

27.- López J, Abarca K, Azócar T. Evidencia clínica y serológica de rickettsiosis canina en Chile. Rev Chil Infectol 2007; 24: 189-93. 